\title{
Electromagnetic Resonances of Silicon Nanoparticle Dimers in the Visible
}

\author{
Urs Zywietz, ${ }^{\dagger, \S}$ Mikolaj K. Schmidt, ${ }^{\ddagger}, \S$ Andrey B. Evlyukhin, ${ }^{\dagger}$ Carsten Reinhardt, ${ }^{\dagger}$ Javier Aizpurua, ${ }^{\ddagger}$ \\ and Boris N. Chichkov* ${ }^{* \dagger}$ \\ ${ }^{\dagger}$ Laser Zentrum Hannover e.V., Hollerithallee 8, D-30419 Hannover, Germany \\ ${ }^{*}$ Centro de Física de Materiales CFM (CSIC-UPV/EHU) and Donostia International Physics Center (DIPC), P. Manuel de \\ Lardizabal 5, 20018 San Sebastián, Spain
}

\section{Supporting Information}

ABSTRACT: We report the optical response of dielectric sub-micrometer particle dimers with resonances in the visible, illustrating a hybridization of electric and magnetic dipolar modes of their individual constituents. The experimental results, corroborated by the numerical calculations, reveal the contributions to the scattering from homogeneous pairs of dipolar electric-electric and magnetic-magnetic modes, as well as from the heterogeneous electric-magnetic modes, induced due to the overlap between the electric and magnetic polarizabilities of single scatterers. The silicon nanoparticles are fabricated on glass by a laser printing method and characterized by polarization-resolved dark-field microscopy. Extensive numerical calculations are carried out to investigate the influence of the morphology and oxidation of the dimers on the optical response in order to properly model their hybridization.

KEYWORDS: nanostructures, hybridization, photonics, silicon, dielectrics, magnetic
$\mathrm{H}$ igh refractive index, sub-micrometer particles have recently emerged as a new building block for tunable, scalable, and low-loss metamaterials. Specifically, it has been shown that spherical silicon nanoparticles provide pronounced resonances associated with the excitation of both magnetic and electric dipolar modes. ${ }^{1-15}$ For a nanoparticle diameter of 100-200 nm these dipolar resonances occur in the visible spectral range. Importantly, the magnetic activity of dielectric nanoparticles is due to the curl of the displacement current and-unlike in plasmonic materials-is not associated with large nonradiative losses. Therefore, such nanoparticles can serve as efficient magnetic scatterers ${ }^{2-10}$ with possible applications as metamaterials, ${ }^{5,16}$ metasurfaces, ${ }^{17}$ nanoantennas, ${ }^{8,10,18-20}$ and novel optical elements exhibiting unusual scattering properties. ${ }^{21,22}$

Recently, it has been suggested that elementary electric and magnetic dipolar resonances of silicon nanospheres can undergo hybridization when interacting in dimer structures, forming bonding and antibonding modes ${ }^{19}$ analogous to those found in plasmonic dimers. ${ }^{23-25}$ However, unlike in the plasmonic dimers, the strong magnetic activity in low-loss structures provides a richer interaction scheme between two magnetic dipolar modes or a heterogeneous pair of electric and magnetic dipolar modes. The exact spectral positions of these hybrid modes depend on the radii and the separation between the nanoparticles forming the dimer. ${ }^{19}$ This dependency has been studied theoretically for nanoparticles providing resonances within the infrared spectral range.

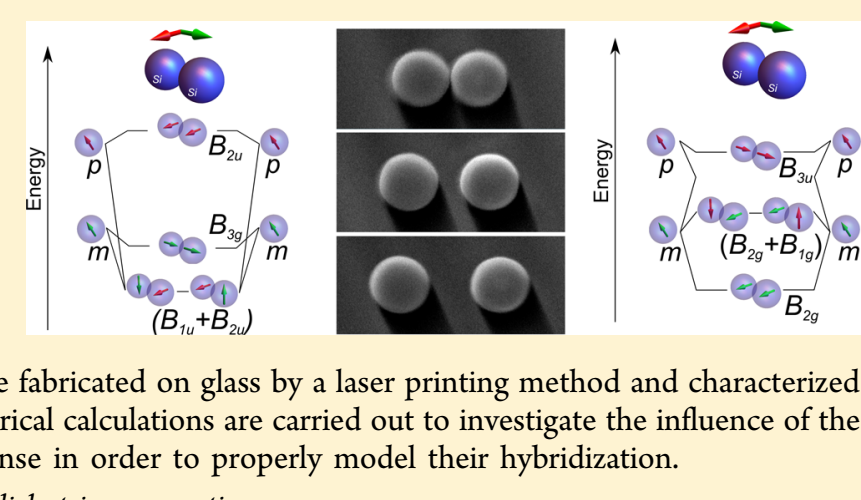

The experimental confirmation of these theoretical predictions was so far hampered by the limitations in methods for the generation of spherical $\mathrm{Si}$ nanoparticles, as well as for depositing them in a controllable fashion on a substrate. Recently, a novel laser printing technique that addresses these problems has been developed. ${ }^{3}$ This technology relies on femtosecond laser printing of $\mathrm{Si}$ nanoparticles onto a glass receiver substrate using silicon-on-insulator (SOI) wafers as laser targets. Nanoparticles generated by this method are initially in the amorphous phase (a-Si), but by additional singlepulse laser irradiation the amorphous nanoparticles can be controllably transformed into crystalline particles (c-Si) or into particles with more complex mixed amorphous/crystalline phases, providing possibilities to tune optical properties of individual Si nanoparticles. The laser printing process is a key technology for the well-controlled generation of more complex dielectric sub-micrometer structures, such as the dimer reported in this work. Alternative approaches for the generation of silicon nanoparticle dimers are possible, for instance with the use of lithographic techniques. ${ }^{26}$ However, the amorphous nature of the samples obtained with this technique makes the optical characterization of the samples very challenging. Femtosecond laser ablation in liquid ${ }^{27}$ can also be used as a fabrication approach, but the limitation in this case relies on the difficulties in obtaining homogeneous constructs for systematic

Received: March 10, 2015 

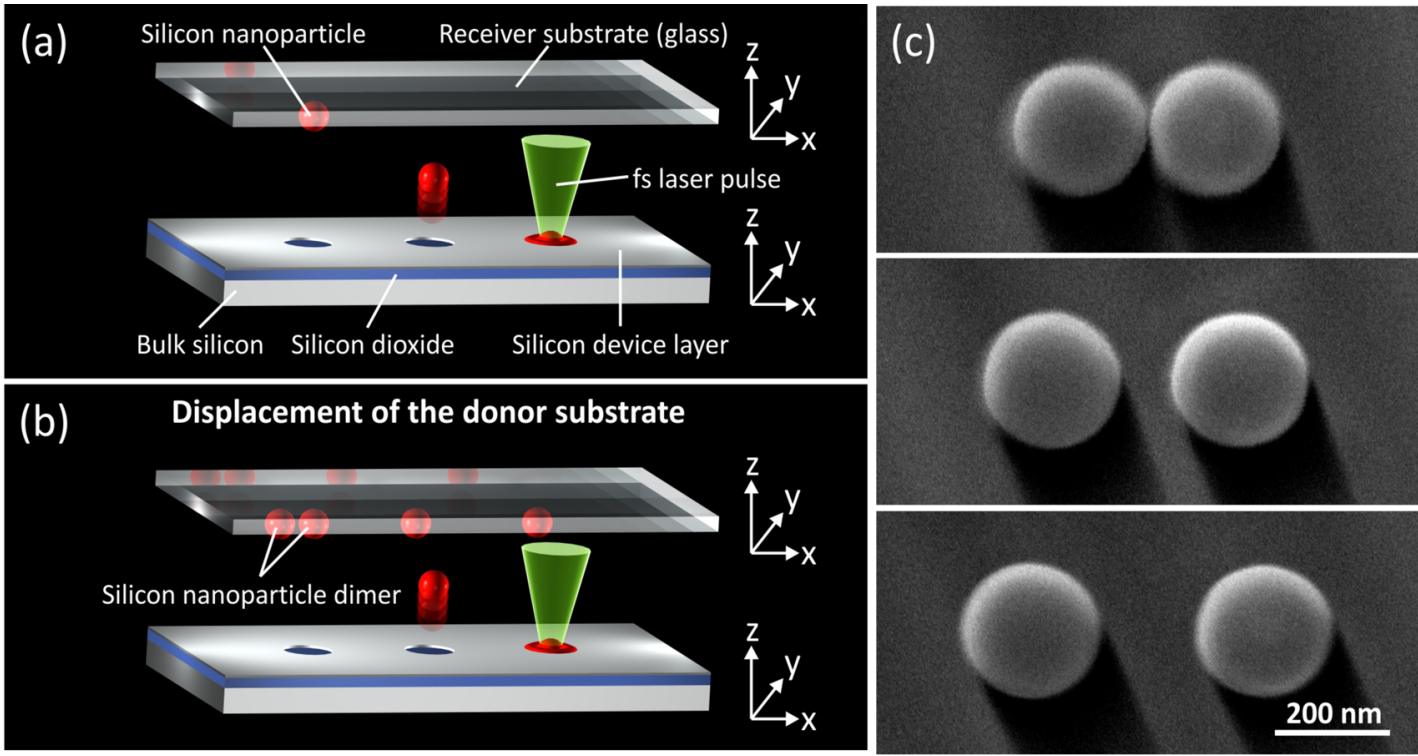

Figure 1. (a) Schematic illustration of femtosecond laser printing of nanoparticles. A silicon-on-insulator (SOI) wafer was used as a target to transfer spherical Si nanoparticles from a $50 \mathrm{~nm}$ crystalline Si layer onto the transparent glass receiver substrate. For printing of Si nanoparticle dimer structures it is possible to translate the target material as well as the receiver substrate independently (b). (c) SEM images of Si nanoparticle dimer structures on a glass substrate.

and controllable studies. Our fabrication approach circumvents these difficulties by generating identical crystalline samples that allow for systematic optical characterization.

We first briefly describe the method of fabrication of the silicon dimer structures and characterize their optical response by means of dark-field scattering spectra. The exact geometry and constitution of each of the $\mathrm{Si}$ nanoparticles are then modeled by means of numerical calculations, and important results concerning the distortion of size and oxidation of the outer layers are reported. Finally, we proceed to the analysis of the dimer structure. In order to interpret the experimental results, we introduce the extended hybridization scheme, which includes both homogeneous electric-electric and magneticmagnetic modes, as well as the novel heterogeneous electricmagnetic modes. These results are corroborated by detailed numerical calculations.

\section{RESULTS}

Dimer Fabrication. For the fabrication of silicon nanoparticle dimer structures, we applied laser printing of nanoparticles from SOI substrates. By using SOI substrates as laser targets it is possible to generate spherical silicon nanoparticles with a very smooth surface and a controllable size. Furthermore, these nanoparticles can be systematically placed and arranged on a receiver substrate. Laser printing does not require any lithographic processes, special operating conditions, or further chemical compounds such as precursors. Different parameters, e.g., laser pulse energy and layer thickness, can be used to influence the resulting nanoparticle diameter. The transfer of nanoparticles from a donor substrate has already been in use for a few years for the generation of metallic nanoparticles. One advanced approach for the generation of single $\mathrm{Si}$ nanoparticles from bulk silicon is the use of a ring-shaped femtosecond laser intensity distribution. ${ }^{28}$ This method is limited to the generation of Si nanoparticles with a diameter as low as $400 \mathrm{~nm}$. The most suitable method to print single Si nanoparticles is to apply SOI wafers as laser targets. Details of the printing process technique, together with the corresponding TEM images addressing the structural properties of the resulting crystallized nanoparticles, can be found in ref 3. A schematic illustration of the generation of nanoparticles and nanoparticle dimer structures is shown in Figure 1. First, silicon nanoparticles are transferred by single femtosecond laser pulse irradiation of an SOI substrate toward a glass receiver substrate (Figure 1a). Subsequently, the SOI substrate is shifted relative to the receiver substrate. By repeating the printing process, silicon nanoparticle dimer structures with varying interparticle distances are realized (Figure 1b). Initially, these structures are in the amorphous phase (a-Si). By additional single-pulse laser irradiation, the amorphous nanoparticles can be controllably transformed into crystalline particles $(\mathrm{c}-\mathrm{Si})$. The resulting silicon nanoparticle dimer structures consist of two identical nanoparticles and are shown in Figure 1c. We estimate the diameters of the nanoparticles to be about $190 \mathrm{~nm}$. However, the exact shape and geometries of the nanoparticles cannot be read out from the SEM images exclusively, as the nanoparticles suffer deformation during the impact against the SOI wafer. Furthermore, as pointed out in refs 2 and 12, the outer layer of silicon oxidizes, forming a $\mathrm{SiO}_{2}$ coating. To address these effects, in section III we present a detailed discussion of how the flattening and oxidation of $\mathrm{Si}$ nanospheres influence the positions of the electric and magnetic dipolar resonances, deriving the most suitable parameters to describe the morphologies of the sample obtained experimentally.

Optical Characterization. Optical characterization of the nanoparticles has been performed with a single nanoparticle spectroscopy setup. Scattered light was measured by the darkfield microscope in transmission mode (see Figure 2). Further information about the scattering setup can be found in ref $3 . \mathrm{Si}$ nanoparticles produced by femtosecond laser printing are initially in the amorphous phase (a-Si). They can be converted into the crystalline phase $(\mathrm{c}-\mathrm{Si})$ by irradiating them with a second femtosecond laser pulse. A similar approach can be applied for the fabrication of a-Si and c-Si dimers. Scattering 


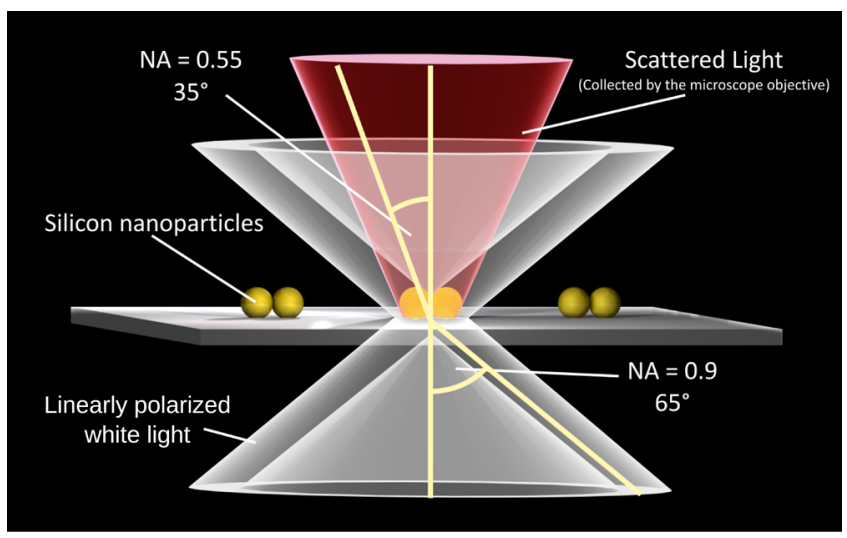

Figure 2. Schematic illustrations of the forward scattering measurements. Linear polarized light is used for illumination. The scattered light of the nanoparticle dimer structure is indicated in red.

spectra of $\mathrm{Si}$ nanoparticle dimers before and after laser crystallization are shown in Figure 3. Scattering resonances of the $\mathrm{c}-\mathrm{Si}$ nanoparticle dimers, compared to those of a-Si nanoparticle dimers, are blue-shifted, and their peak intensities increased. Such behavior is explained by the changes of the silicon dielectric function during the crystallization of silicon.

We also note that the spectral positions of resonances in Figure 3 depend on the distance between the nanoparticles of the dimer, indicating hybridization of the localized modes. To study this effect in detail, we have performed polarizationresolved measurements. In Figure 4 we show spectra of the dimer structures with a separation of $375 \mathrm{~nm}(\mathrm{a}, \mathrm{b})$ and $5 \mathrm{~nm}$ (c, d), illuminated by light polarized perpendicularly $(a, c)$ or parallelly $(b, d)$ to the long axis of the dimer. Measurements of the $\mathrm{Si}$ nanoparticle dimer structure with an interparticle distance of about $375 \mathrm{~nm}$ show no significant difference in the spectral position of the scattering peaks for different polarizations (see Figure $4 \mathrm{a}$ and $\mathrm{b}$ ), indicating a weak electromagnetic interaction between the nanoparticles in dimers. In contrast, scattering measurements for the case of a small interparticle distance $(5 \mathrm{~nm})$ demonstrate a significant difference between the two polarizations (see Figure $4 c$ and $d$ ). For light polarized parallelly to the major dimer axis a single broad scattering peak emerges (Figure 4d) between the two resonant features observed for the perpendicular polarization (Figure 4c).

\section{ANALYSIS OF THE OPTICAL RESPONSE}

In order to understand the optical response of the dimer structures shown in Figure 4 and to derive the geometric properties of the individual scatterers, in the following sections we model their optical response with the FDTD method. ${ }^{29}$ First, we focus on the simpler system of a single nanoparticle to explore important effects that determine the optical response of these structures: the oxidation of the outer layer of silicon, ${ }^{2,12}$ the influence of the substrate, the distortion of the shape of the scatterer, and finally, the dark-field illumination scheme. We then proceed to analyzing the optical response of dimer structures and its dependence on the separation between the scatterers and on the polarization of the incident light. The optical constants for crystalline silicon are taken from the literature. $^{30}$

Single Scatterers. The optical spectrum of a single c-Si scatterer can be influenced by many different factors such as the presence of the substrate, the oxidation of the particle, or a departure from the spherical shape of the particle. We analyze all these effects in connection with the spectra of noninteracting nanoparticles shown in Figure $3 \mathrm{~b}$ to determine which morphology of particles reproduces the fine details of the spectra. As we will show below, the analysis of the different factors affecting the optical response of single c-Si particles is crucial to correctly interpret their spectral features, as well as to separate the effects of hybridization in dimer structures from purely morphological effects.

Presence of the Substrate. The black solid line in Figure 5a denotes the scattering cross section of a c-Si nanoparticle of 97 $\mathrm{nm}$ radius in air, with two dominant contributions: electric and magnetic dipolar modes marked with the green and red lines, respectively. As previously reported, ${ }^{2}$ the spectral positions of lowest order Mie resonances in dielectric spheres are rather insensitive to the presence of substrate (dashed line). This is mostly due to the localization of the displacement currents inside the dielectric material. We observe that effect in Figure $5 \mathrm{a}$, where both the dipolar electric and magnetic resonances of a $97 \mathrm{~nm}$ radius $\mathrm{c}-\mathrm{Si}$ nanosphere peak at 600 and $750 \mathrm{~nm}$ independently of the presence of a silica substrate.

Oxidation of the Particle. Silicon nanoparticles undergo oxidation of their outer layers, a process that leads to the formation of a core-shell nanoparticle with a shrunken c-Si core and a silica shell. Since the refractive index of silica is significantly lower than that of the $\mathrm{c}-\mathrm{Si}$, the optical response of the nanoparticle is primarily determined by the c-Si core. In Figure $5 b$ we show how the optical features blue-shift with the shell thickness $h$ increasing from $0 \mathrm{~nm}$ (black line) to $8 \mathrm{~nm}$
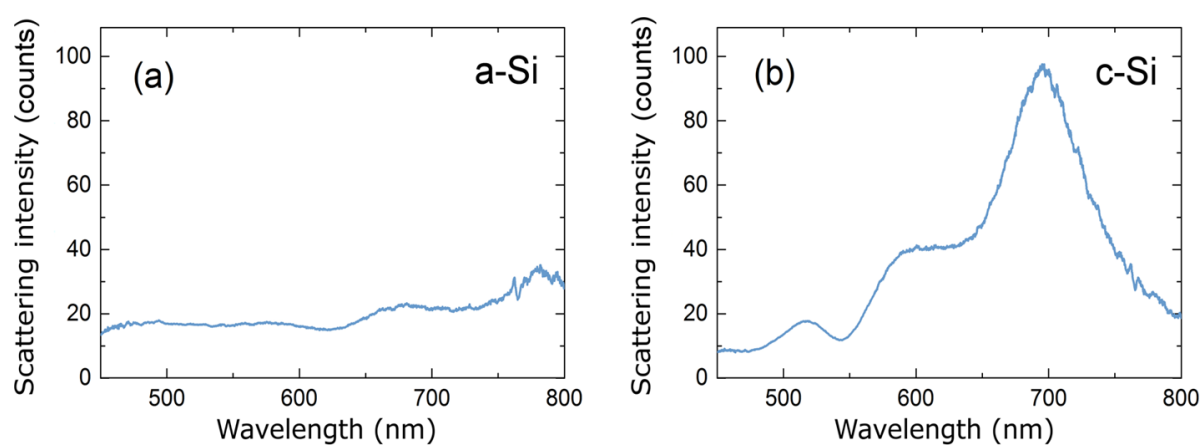

Figure 3. Scattering spectra of (a) amorphous silicon a-Si and (b) crystalline silicon c-Si dimer structures. Measurements were carried out with unpolarized light. 

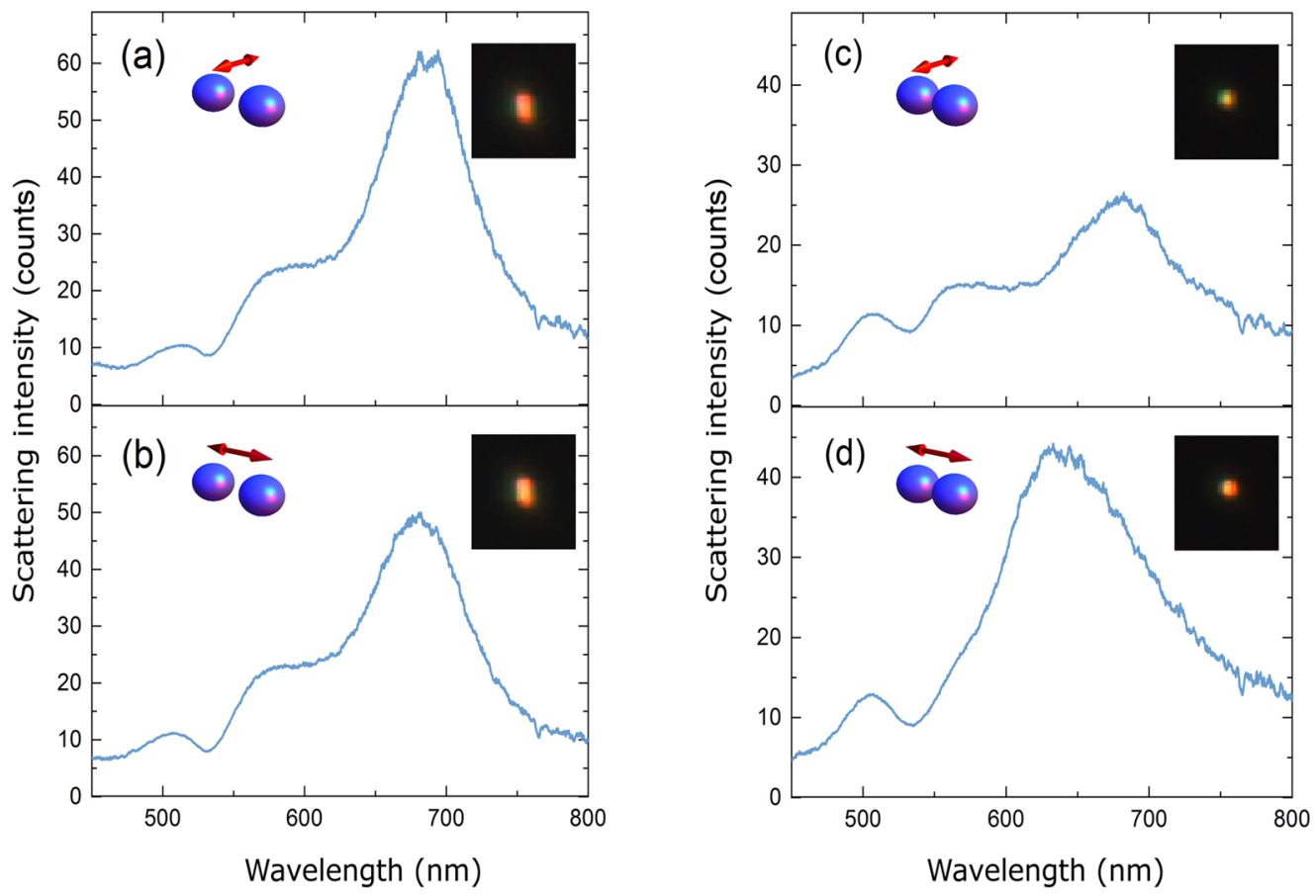

Figure 4. Scattering spectra of crystalline silicon nanoparticle dimer structures for incident light polarized perpendicularly (a, c) and parallelly (b, d) to the axis of the dimer structure. The distance between the nanoparticle surfaces is $375 \mathrm{~nm}(\mathrm{a}, \mathrm{b})$ and $5 \mathrm{~nm}(\mathrm{c}$, d) and was measured using SEM image analysis software with the standard deviation of $0.8 \mathrm{~nm}$. Insets demonstrate the dark scattering images of the corresponding dimer structures.

(blue line) and simultaneously decreasing the core radius from $97 \mathrm{~nm}$ to $89 \mathrm{~nm}$.

Distorting the Shape of a Nanoparticle. Silicon nanoparticles formed in the printing process are not ideally spherical, but rather take the form of oblate spheroids. As shown in Figure 5c, such lowering of the symmetry of the scatterers influences the electric and magnetic dipolar modes slightly differently. Specifically, contracting the nanoparticle along the illumination direction (changing $r_{z}$ ) shifts the lowenergy magnetic and high-energy electric modes by 70 and 20 $\mathrm{nm}$, respectively. This is clearly a very different behavior than the homogeneous shift induced by the formation of the oxide layer. A similar analysis of the geometric parameters of nanodisks and their effect on the optical response can be found in ref 8 .

Angled Incidence. To model the dark-field scattering microscopy scheme, we present the spectra obtained with varying illumination angles. As observed in Figure 5d, changing the angle of illumination modifies the relative intensity of the resonances. For instance, it is possible to eliminate the scattering from the $525 \mathrm{~nm}$ mode (quadrupolar magnetic) for grazing incidence.

Considering all the effects reported above, we arrive at the optimal parameters that correctly describe the spectral features of the optics of the single scatterers prepared by laser printing. We will hence describe the nanoparticles as ellipsoidal core(c$\mathrm{Si})-\operatorname{shell}\left(\mathrm{SiO}_{2}\right)$ structures with major and minor core radii of 95 and $78 \mathrm{~nm}$, respectively, and a $4 \mathrm{~nm}$ oxide layer.

On comparing the measured spectra of dimers shown in Figure $4 \mathrm{a}, \mathrm{b}$ with the scattering cross section calculated for a single spherical Si nanoparticle presented in Figure 5a, one can see that the two main resonances at wavelengths of 675 and $575 \mathrm{~nm}$ in the experimental spectra correspond to the excitation of magnetic and electric dipole modes, respectively. However, in contrast to the theoretical spectrum in Figure 5a, an additional quadrupolar resonance peak is present in the experimental curves at a wavelength of approximately $510 \mathrm{~nm}$ independently of interparticle distance in the dimers (Figure 4). As shown in Figure 5c, this additional resonance strengthens as a result of shape deformation (from sphere to oblate spheroid) of $\mathrm{Si}$ nanoparticles in the fabrication process. Indeed, for an oblate ellipsoid, the scattering cross sections presented in Figure $5 \mathrm{c}, \mathrm{d}$ have an additional resonance near the wavelength $(510 \mathrm{~nm})$ of the experimental realization. Note that the intensity of scattering at this resonance strongly depends on the direction of the incident light.

Dimer Structures. Once we have understood the effects of morphology of single c-Si scatterers, we can safely turn to the analysis of the electromagnetic coupling in more complex structures. The optical response of dimer structures shown in Figures 3 and 4 can be understood through the hybridization of modes excited in the single constituent dielectric scatterers. The formation of dimer modes, reminiscent of that observed in numerous plasmonic systems, occurs between the elementary electric and magnetic dipolar modes of the scatterers, as shown in Figures 6 and 7, where we analyze separately the cases of normally incident light with electric field (denoted as a red arrow) polarized along and perpendicularly to the major dimer axis, respectively. We neglect here the interaction between higher order modes, as their electromagnetic fields are largely confined inside the dielectric, and discard the asymmetry introduced into the system by the incident light. Within these approximations, we can consider only the modes of the dimer that transform according to particular irreducible representations of the $D_{2 h}$ dimer symmetry group. ${ }^{25,31}$ Labels of the relevant representations are shown in the schematics of Figures 6 and 7. The spectra shown in these figures have been calculated for free-standing systems comprising nanoparticles with shape and structure as discussed above (see the 
(a)
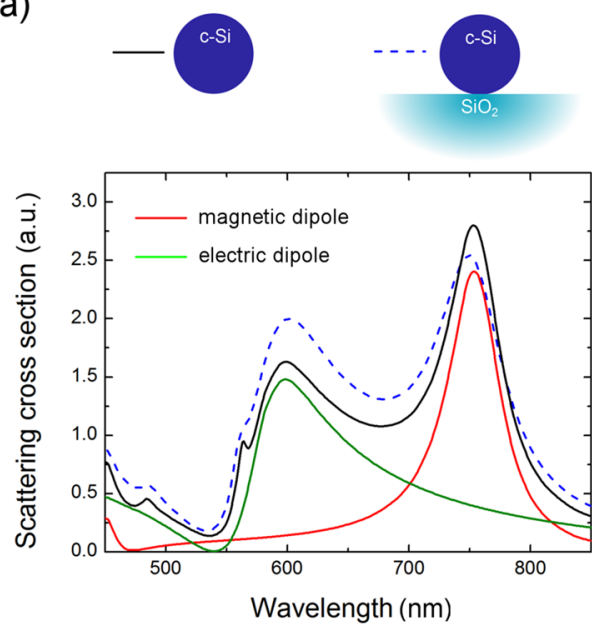

(c)

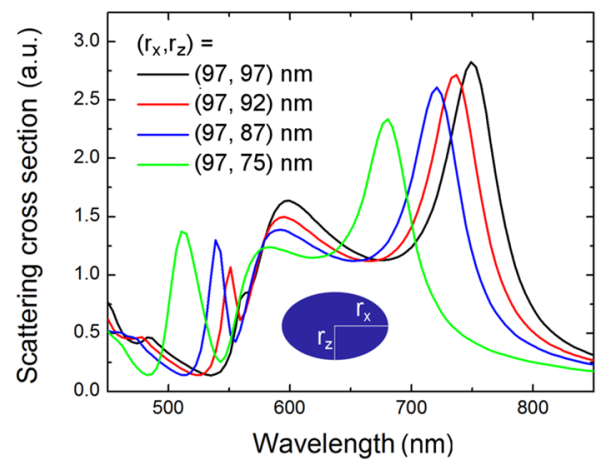

(b)
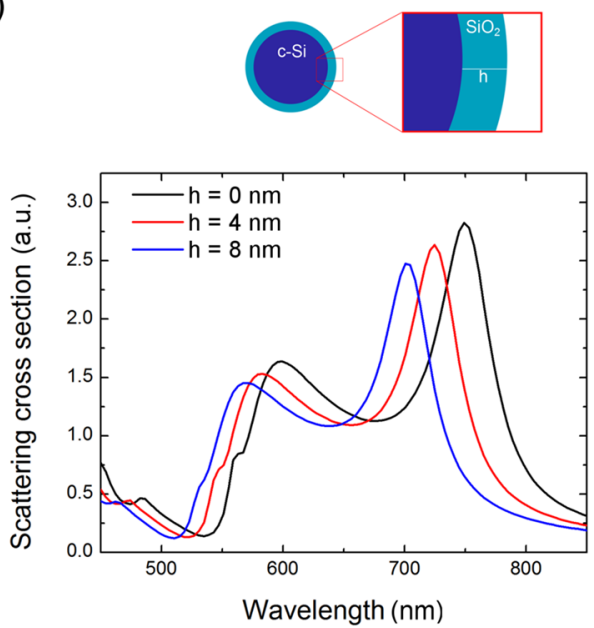

(d)

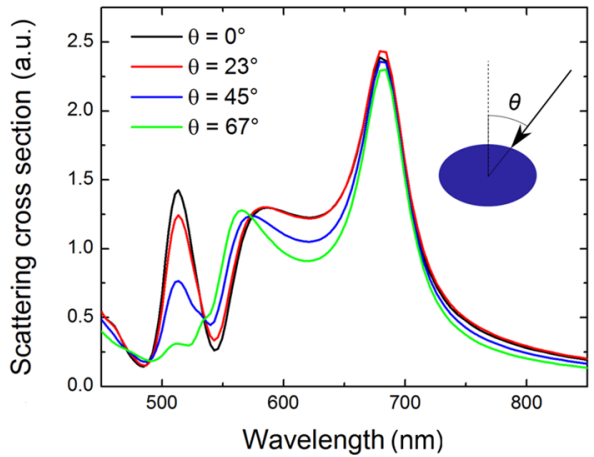

Figure 5. Calculated scattering cross sections of single dielectric scatterers. (a) Scattering by a c-Si nanosphere of 97 nm radius (black solid line) is dominated by the electric dipolar (green line) and magnetic dipolar (red line) contributions. Spectral positions of these resonances are largely insensitive to the presence of the silica substrate (dashed line). (b) Oxidation of the outer layer of the nanoparticle of thickness $h$ significantly blueshifts all of the spectral features. (c) Contraction of the c-Si scatterer along the illumination direction ( $z$-axis) selectively blue-shifts magnetic resonances. (d) By modifying the illumination angle, we can selectively tune the strengths of higher order resonances. Here, calculations for the oblate spheroid described by $\left(r_{x}, r_{z}\right)=(97,75) \mathrm{nm}$ are shown.

Supporting Information for discussion of the angled illumination and the effects of the substrate).

Let us first consider the modes excited by the light polarized along the major axis of the dimer. In the hybridization scheme shown in Figure 6 a we reveal the symmetries of the single nanoparticle dipolar modes, denoted by red and green arrows for electric and magnetic dipoles, respectively. The two homogeneous modes denoted as $\mathrm{B}_{3 \mathrm{u}}$ (so-called electric bonding mode) and $\mathrm{B}_{2 \mathrm{~g}}$ couple directly to the incident light, as they exhibit nonvanishing electric and magnetic dipolar modes, respectively, in the polarization plane. In Figure $6 \mathrm{~b}$ we present the experimental (black lines) and calculated (solid red lines) scattering intensities of dimer structures for varying dimer separations $d$ ranging from $320 \mathrm{~nm}$ (top) to $5 \mathrm{~nm}$ (bottom plot). For each spectrum we have also calculated the strengths of the dipoles induced in each of the nanoparticles $\left(\mathbf{p}_{i}, \mathbf{m}_{i}\right)$ by integrating the polarization $\mathbf{P}\left(\mathbf{p}_{i}=\int_{V_{i}} \mathbf{P} \mathrm{d} V\right)$ and polarization currents $\mathbf{J}\left(\mathbf{m}_{i}=(1 / 2) \int_{V_{i}} \mathbf{r} \times \mathbf{J} \mathrm{d} V\right)$ inside the volume of the dielectric $V_{i}$. Having obtained these quantities, we have calculated the contribution to the scattering from the different modes. For example, the scattering from the bonding mode $B_{3 u}$ (dashed green line contribution) is dominant for all the dimer separations $d$, and it red-shifts with decreasing $d$, similarly to the electric bonding modes of plasmonic dimer antennas (see the
Supporting Information for details on calculating the contribution from each mode). Simultaneously, the magnetic mode $\mathrm{B}_{2 \mathrm{~g}}$, denoted with a dashed orange line, blue-shifts and decreases significantly in intensity. We note that this shift is not monotonous and might point to a more complex near-field interaction not described within our model. Finally, we consider the two degenerated heterogeneous modes (marked collectively in the hybridization scheme as $\left(B_{2 g}+B_{1 g}\right)$ ), each comprising one electric and one magnetic dipole. These modes are induced indirectly by the incident light coupling to the magnetic dipolar mode of one sphere, which in turn induces the electric dipole in the other sphere. Since these two hybrid modes are induced coherently, they lead to the effective excitation of both magnetic $B_{2 g}$ and electric $B_{1 g}$ modes. ${ }^{19}$ This excitation mechanism is confirmed by tracing the scattering contribution from mode $B_{1 g}$ (dashed blue line), which follows the magnetic mode $\mathrm{B}_{2 \mathrm{~g}}$, blue-shifting with decreasing separation d.

We note that our calculations reveal a remarkable correlation with the experimental results (see comparison in Figure $6 \mathrm{~b}$ ). Importantly, and somewhat unexpectedly, we observe a small contribution to the scattering from the heterogeneous modes, comprising electric and magnetic dipolar excitation, in the experimental results around $570 \mathrm{~nm}$ for a $d=5 \mathrm{~nm}$ separation. The disagreement in the intensities of the features most likely 

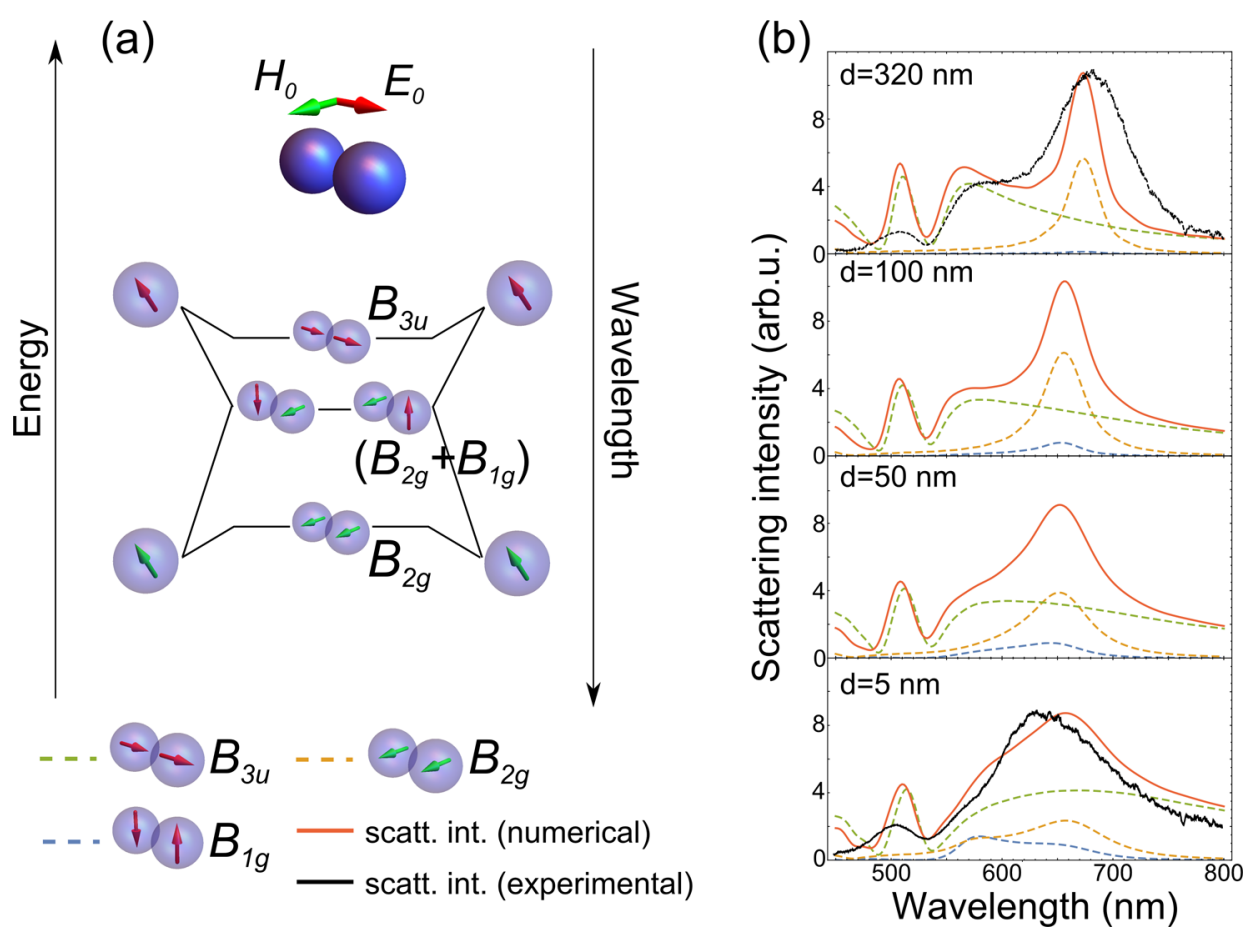

Figure 6. Scattering of normally incident light polarized along the axis of a dimer of silicon scatterers. (a) Energy-level diagram describing the hybridization of electric (red arrows) and magnetic (green arrows) dipolar resonances of single scatterers with the representations of $D_{2 h}$ symmetry group: $\mathrm{B}_{3 \mathrm{u}}, \mathrm{B}_{1 \mathrm{~g}}$, and $\mathrm{B}_{2 \mathrm{~g}}$. (b) Calculated scattering intensities of dimer structures (solid red line) for separations of $d=320,100,50$, and $5 \mathrm{~nm}$ (top to bottom) compared to the experimental results (black line). The spectra are decomposed according to the hybridization scheme of electric and magnetic modes: $\mathrm{B}_{3 \mathrm{u}}$ (green dashed line), $\mathrm{B}_{1 \mathrm{~g}}$ (blue dashed line), and $\mathrm{B}_{2 \mathrm{~g}}$ (orange dashed line). Each single scatterer is an oblate ellipsoidal core(c$\mathrm{Si})-\operatorname{shell}\left(\mathrm{SiO}_{2}\right)$ structure with major and minor external radii of 95 and $78 \mathrm{~nm}$, respectively, and a 4 nm oxide layer.

(a)

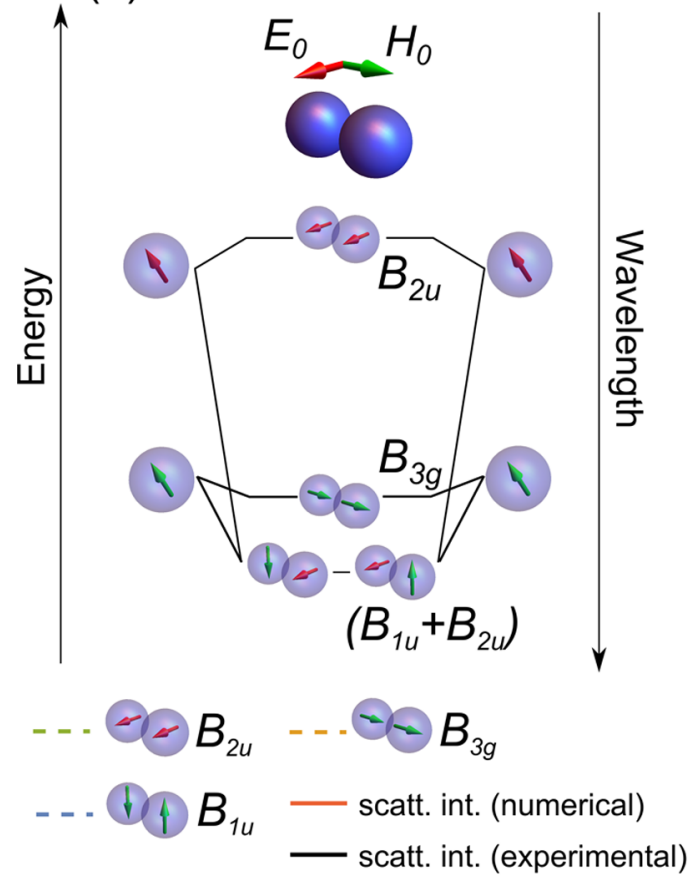

(b)

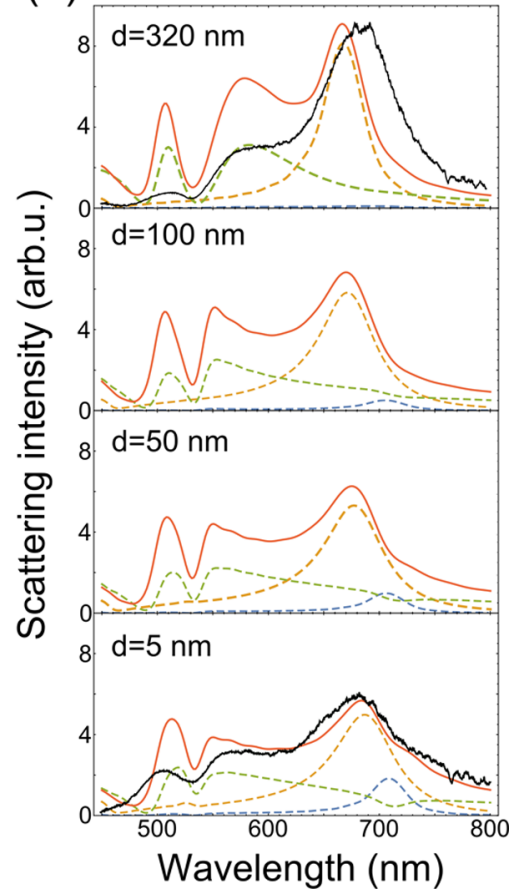

Figure 7. Scattering of normally incident light polarized perpendicularly to the axis of a dimer of silicon scatterers. (a) Energy-level diagram describing the hybridization of electric (red arrows) and magnetic (green arrows) dipolar resonances of single scatterers with the representations of $D_{2 h}$ symmetry group: $\mathrm{B}_{2 w}, \mathrm{~B}_{3 g}$, and $\mathrm{B}_{1 \mathrm{u}}$. (b) Calculated scattering intensities of dimer structures (solid red line) for separations of $d=320,100,50$, and $5 \mathrm{~nm}$ (top to bottom) compared to the experimental results (black line). The spectra are decomposed according to the hybridization scheme of electric and magnetic modes: $\mathrm{B}_{2 \mathrm{u}}$ (green dashed line), $\mathrm{B}_{1 \mathrm{u}}$ (blue dashed line), and $\mathrm{B}_{3 \mathrm{~g}}$ (orange dashed line). Scatterers are identical to those used in Figure 6. 
stems from the setup of the dark-field microscope, i.e., the finite aperture and the non-normal incidence of light.

We have conducted a similar analysis for the TE polarization of the incident light (electric field normal to the dimer axis). The hybridization scheme shown in Figure 7 reveals the emergence of two homogeneous modes, electric $B_{2 u}$ and bonding magnetic $\mathrm{B}_{3 g}$, both induced directly by the incident light. We also consider the heterogeneous mode composed as the combination of electric and magnetic single-particle excitations. This last mode is excited due to the coupling between the electric dipole induced by the incident light and the magnetic dipole of the adjoining sphere in a manner analogous to the heterogeneous mode discussed in Figure 6, but reversing the role of electric and magnetic dipoles. Effectively, the pair of these degenerate heterogeneous modes gives rise to the excitation of the magnetic $B_{1 u}$ mode. The decomposition of the scattering intensity in Figure $7 \mathrm{~b}$ reveals the evolutions of the modes as indicated by the hybridization scheme (Figure 7a), with the red-shifting $B_{1 \mathrm{u}}$ (dashed blue) and $\mathrm{B}_{3 \mathrm{~g}}$ (dashed orange) and blue-shifting $\mathrm{B}_{2 \mathrm{u}}$ (dashed green) modes. As in the previous case, we observe a very good agreement between the experimental and numerical results, including a hint of the heterogeneous mode at around $710 \mathrm{~nm}$ for the smallest separation (blue dashed line contribution).

Finally, we note that the additional resonant feature at the wavelength of $510 \mathrm{~nm}$ (see Figures 6 and 7) is weakly dependent on the separation of the dimers for both polarizations of light, further pointing to the multipolar character of this excitation.

Finally, we stress that in all the calculations shown above we have only considered normally incident excitation and therefore limited ourselves to accounting for modes that can be excited in such a highly symmetric setup. Nevertheless, our numerical results reproduce all the dominant features of the experimental dark-field scattering spectra measured with a highly asymmetric setup shown in Figure 2. This agreement indicates that all the dimer modes that were dark under normally incident light either are not excited in the dark-field setup or do not contribute to the scattering spectra. We present a more detailed study of these dark modes and their excitation mechanism under grazing incidence of light in the Supporting Information.

\section{CONCLUSION}

We have investigated dimers of sub-micrometer crystalline silicon nanoparticles with different interparticle distances, ranging from 5 to $375 \mathrm{~nm}$. The dimers have been fabricated on a glass substrate by the laser printing method, which has been recently developed for $\mathrm{Si}$ nanoparticles with resonant optical responses. We have experimentally demonstrated that the resonant optical behavior of the dimers is dependent on the interparticle distance and the polarization of the incident light. For small interparticle distances the electric and magnetic dipolar modes of single scatterers hybridize and form homogeneous electric-electric, magnetic-magnetic, and more complex heterogeneous electric-magnetic modes, following the hybridization scheme discussed herein. We note that the magnetic activity of the dielectric nanoparticles promotes a much richer hybridization scheme than that observed in the dimers of plasmonic nanoparticles, and it requires further investigation. These findings are reproduced very accurately by numerical simulations, which further allowed us to access information about the exact shape and composition of the scatterers. We conclude that the tunable and strongly interacting dielectric dimers are a versatile tool for studying the light coupling in dielectric nanostructures. Furthermore, as building blocks for more complex systems, they carry the potential to replace lossy plasmonic gap nanoantennas as a robust platform to host a variety of field-enhanced spectroscopies, such as SERS and SEIRA, and lead to novel, advanced metasurfaces.

\section{ASSOCIATED CONTENT}

\section{S Supporting Information}

Calculations of the scattering intensities of hybridized modes under tilted illumination. Effects of the oblateness of nanoparticles and the presence of substrate and an oxide layer on the scattering spectra of dimers. The Supporting Information is available free of charge on the ACS Publications website at DOI: 10.1021 /acsphotonics.5b00105.

\section{AUTHOR INFORMATION}

\section{Corresponding Author}

*E-mail: b.chichkov@lzh.de.

\section{Author Contributions}

${ }^{\S}$ U. Zywietz and M. K. Schmidt contributed equally.

\section{Notes}

The authors declare no competing financial interest.

\section{ACKNOWLEDGMENTS}

The authors would like to thank Dr. Aitzol Garcia-Etxarri for fruitful discussions. The authors acknowledge financial support of this work from Deutsche Forschungsgemeinschaft (DFG) (SFB/TRR 123 "PlanOS"). M.K.S. and J.A. acknowledge funding from the project FIS2013-41184-P of the Spanish Ministry of Economy and Competitiveness, the ETORTEK IE14-393 NANOGUNE'14 project of the Department of Industry of the Government of the Basque Country, project IT756-13 of the Department of Education and Culture of the Basque Country, and scholarship AP-2012-4204 from the Spanish Ministry of Education, Culture and Sport. A.B.E. and B.N.C. acknowledge support from the Ministry of Education and Science of Russian Federation (project 14.B25.31.0019).

\section{REFERENCES}

(1) Evlyukhin, A. B.; Reinhardt, C.; Seidel, A.; Luk'yanchuk, B. S.; Chichkov, B. N. Optical response features of Si-nanoparticle arrays. Phys. Rev. B 2010, 82, 045404.

(2) Evlyukhin, A. B.; Novikov, S. M.; Zywietz, U.; Eriksen, R. L.; Reinhardt, C.; Bozhevolnyi, S. I.; Chichkov, B. N. Demonstration of magnetic dipole resonances of dielectric nanospheres in the visible region. Nano Lett. 2012, 12, 3749-3755.

(3) Zywietz, U.; Evlyukhin, A. B.; Reinhardt, C.; Chichkov, B. N. Laser printing of silicon nanoparticles with resonant optical electric and magnetic responses. Nat. Commun. 2014, 5, 3402.

(4) Garcia-Etxarri, A.; Gomez-Medina, R.; Froufe-Perez, L.; Lopez, C.; Chantada, L.; Scheffold, F.; Aizpurua, J.; Nieto-Vesperinas, M.; Saenz, J. Strong magnetic response of submicron silicon particles in the infrared. Opt. Express 2011, 19, 4815-4826.

(5) Zhao, Q.; Zhou, J.; Zhang, F.; Lippens, D. Mie resonance-based dielectric metamaterials. Mater. Today 2009, 12, 60-69.

(6) Gómez-Medina, R.; García-Cámara, B.; Suárez-Lacalle, I.; González, F.; Moreno, F.; Nieto-Vesperinas, M.; Sáenz, J. J. Electric and magnetic dipolar response of germanium nanospheres: interference effects, scattering anisotropy, and optical forces. J. Nanophotonics 2011, 5, 053512 . 
(7) Krasnok, A. E.; Miroshnichenko, A. E.; Belov, P. A.; Kivshar, Y. S. All-dielectric optical nanoantennas. Opt. Express 2012, 20, 2059920604.

(8) Staude, I.; Miroshnichenko, A. E.; Decker, M.; Fofang, N. T.; Liu, S.; Gonzales, E.; Dominguez, J.; Luk, T. S.; Neshev, D. N.; Brener, I.; Kivshar, Y. Tailoring directional scattering through magnetic and electric resonances in subwavelength silicon nanodisks. ACS Nano 2013, 7, 7824-7832.

(9) García-Cámara, B.; Gómez-Medina, R.; Sáenz, J. J.; Sepúlveda, B. Sensing with magnetic dipolar resonances in semiconductor nanospheres. Opt. Express 2013, 21, 23007-23020.

(10) Schmidt, M. K.; Esteban, R.; Saenz, J. J.; Suárez-Lacalle, I.; Mackowski, S.; Aizpurua, J. Dielectric antennas - a suitable platform for controlling magnetic dipolar emission. Opt. Express 2012, 20, 1363613650 .

(11) Kuznetsov, A. I.; Miroshnichenko, A. E.; Fu, Y. H.; Zhang, J.; Luk'yanchuk, B. Magnetic light. Sci. Rep. 2012, 2, 492.

(12) Fu, Y. H.; Kuznetsov, A. I.; Miroshnichenko, A. E.; Yu, Y. F.; Luk'yanchuk, B. Directional visible light scattering by silicon nanoparticles. Nat. Commun. 2013, 4, 1527.

(13) Yang, Y.; Wang, W.; Moitra, P.; Kravchenko, I. I.; Briggs, D. P.; Valentine, J. Dielectric meta-reflectarray for broadband linear polarization conversion and optical vortex generation. Nano Lett. 2014, 14, 1394-1399.

(14) Habteyes, T. G.; Staude, I.; Chong, K. E.; Dominguez, J.; Decker, M.; Miroshnichenko, A.; Kivshar, Y.; Brener, I. Near-field mapping of optical modes on all-dielectric silicon nanodisks. ACS Photonics 2014, 1, 794-798.

(15) Zou, L.; Withayachumnankul, W.; Shah, C. M.; Mitchell, A.; Bhaskaran, M.; Sriram, S.; Fumeaux, C. Dielectric resonator nanoantennas at visible frequencies. Opt. Express 2013, 21, 1344-1352.

(16) Shi, L.; Tuzer, T. U.; Fenollosa, R.; Meseguer, F. A new dielectric metamaterial building block with a strong magnetic response in the sub-1.5-micrometer region: silicon colloid nanocavities. Adv. Mater. 2012, 24, 5934-5938.

(17) Yu, N.; Capasso, F. Flat optics with designer metasurfaces. Nat. Mater. 2014, 13, 139-150.

(18) Rolly, B.; Stout, B.; Bonod, N. Boosting the directivity of optical antennas with magnetic and electric dipolar resonant particles. Opt. Express 2012, 20, 20376-20386.

(19) Albella, P.; Poyli, M. A.; Schmidt, M. K.; Maier, S. A.; Moreno, F.; Saáenz, J. J.; Aizpurua, J. Low-loss electric and magnetic fieldenhanced spectroscopy with subwavelength silicon dimers. J. Phys. Chem. C 2013, 117, 13573-13584.

(20) Staude, I.; Khardikov, V. V.; Fofang, N. T.; Liu, S.; Decker, M.; Neshev, D. N.; Luk, T. S.; Brener, I.; Kivshar, Y. S. Shaping photoluminescence spectra with magnetoelectric resonances in alldielectric nanoparticles. ACS Photonics 2015, 2, 172-177.

(21) Schmidt, M. K.; Aizpurua, J.; Zambrana-Puyalto, X.; Vidal, X.; Molina-Terriza, G.; Sáenz, J. J. Isotropically polarized speckle patterns. Phys. Rev. Lett. 2015, 114, 113902.

(22) García-Etxarri, A.; Dionne, J. A. Surface-enhanced circular dichroism spectroscopy mediated by nonchiral nanoantennas. Phys. Rev. B 2013, 87, 235409.

(23) Nordlander, P.; Oubre, C.; Prodan, E.; Li, K.; Stockman, M. I. Plasmon hybridization in nanoparticle dimers. Nano Lett. 2004, 4, 899-903.

(24) Romero, I.; Aizpurua, J.; Bryant, G. W.; García De Abajo, F. J. Plasmons in nearly touching metallic nanoparticles: singular response in the limit of touching dimers. Opt. Express 2006, 14, 9988-9999.

(25) Hopkins, B.; Poddubny, A. N.; Miroshnichenko, A. E.; Kivshar, Y. S. Revisiting the physics of Fano resonances for nanoparticle oligomers. Phys. Rev. A 2013, 88, 053819.

(26) Bakker, R. M.; Permyakov, D.; Yu, Y. F.; Markovich, D.; Paniagua-Domínguez, R.; Gonzaga, L.; Samusev, A.; Kivshar, Y.; Lukyanchuk, B.; Kuznetsov, A. I. Magnetic and electric hotspots with silicon nanodimers. Nano Lett. 2015, 15, 2137-2142.
(27) Yan, J.; Liu, P.; Lin, Z.; Wang, H.; Chen, H.; Wang, C.; Yang, G. Directional Fano resonance in a silicon nanosphere dimer. ACS Nano 2015, 9, 2968-2980.

(28) Zywietz, U.; Reinhardt, C.; Evlyukhin, A. B.; Birr, T.; Chichkov, B. N. Generation and patterning of Si nanoparticles by femtosecond laser pulses. Appl. Phys. A: Mater. Sci. Process. 2014, 114, 45-50.

(29) Taflove, A. Application of the finite-difference time-domain method to sinusoidal steady-state electromagnetic-penetration problems. IEEE Trans. Electromagn. Compat. 1980, EMC-22, 191-202.

(30) Palik, E. D. Handbook of Optical Constants of Solids; Academic Press, 1997.

(31) The $D_{2 h}$ symmetry group describes the simplified system, which does not account for the asymmetry introduced by the incident light. For example, we do not distinguish between the $B_{1 g}$ modes induced directly by the TE-polarized light and the $\mathrm{B}_{1 \mathrm{~g}}$ modes indirectly excited in the TM polarization. In the two cases the electric dipoles comprising those modes are oriented either perpendicularly or parallelly to the wavevector of the incident light, respectively. 